\title{
Soft drinks consumption, diet quality and BMI in a Mediterranean population
}

\author{
Eva Balcells ${ }^{1}$, Mario Delgado-Noguera ${ }^{2}$, Ricardo Pardo-Lozano ${ }^{3}$, \\ Taïs Roig-González ${ }^{4}$, Anna Renom ${ }^{4}$, Griselda González-Zobl ${ }^{5}$, Juan Muñoz-Ortego ${ }^{6}$, \\ Susana Valiente-Hernández ${ }^{7}$, Marina Pou-Chaubron ${ }^{8}$ and Helmut Schröder $9,10, *$ \\ ${ }^{1}$ Servei de Pneumologia, Hospital del Mar - IMIM, Barcelona, Spain: ${ }^{2}$ Departaments of Obstetrics and \\ Gynecology and Preventive Medicine, Universidad Autónoma de Barcelona, Barcelona, Spain: ${ }^{3}$ Grupo de \\ Investigación Clínica en Farmacología Humana y Neurociencias, Hospital del Mar - IMIM, Barcelona, Spain: \\ ${ }^{4}$ Department of Geriatrics, Institut Municipal d'Assistència Sanitària (IMAS), Barcelona, Spain: ${ }^{5}$ Unitat Docent \\ de Medicina Preventiva i Salut Pública IMAS-UPF-ASPB, Barcelona, Spain: 'Servicio de Reumatología, \\ Hospital del Mar - IMIM, Barcelona, Spain: ${ }^{7}$ Centro de Salud Ramón Turró de Barcelona, Barcelona, Spain: \\ ${ }^{8}$ Unidad de Medicina Física y Rehabilitación, Fundació Hospital Asil de Granollers, Granollers, Spain: \\ ${ }^{9}$ Cardiovascular Risk and Nutrition Research Group (CARINULEC), Municipal Institute of Medical Research \\ (IMIM - Hospital del Mar), Biomedical Research Park (Parc de Recerca Biomèdica de Barcelona - PRBB), \\ c/Doctor Aigaunder 88, 08003 Barcelona, Spain: ${ }^{10} \mathrm{CIBER}$, Fisiopatología de la Obesidad y Nutrición \\ (CIBEROBN), Madrid, Spain
}

Submitted 14 January 2010: Accepted 31 August 2010: First published online 19 October 2010

\begin{abstract}
Objectives: Evidence of the effects of soft drinks consumption on BMI and lifestyle in adult populations is mixed and quite limited. The aim of the present study was to determine the association of soft drinks consumption with BMI and lifestyle in a representative Mediterranean population.

Design: Two independent, population-based, cross-sectional (2000 and 2005) studies. Dietary intake was assessed using a validated FFQ. Weight and height were measured.

Setting: Girona, Spain.

Subjects: Random sample of the 35- to 74-year-old population (3910 men and 4285 women).

Results: Less than half $(41.7 \%)$ of the population consumed soft drinks; the mean consumption was $36 \cdot 2 \mathrm{ml} / \mathrm{d}$. The prevalence of sedentary lifestyle increased with the frequency of soft drinks consumption $(P=0 \cdot 025)$. Daily soft drinks consumption significantly increased the risk of low adherence to the Mediterranean diet $(\mathrm{OR}=0 \cdot 57,95 \% \mathrm{CI} 0 \cdot 44,0 \cdot 74 v$. top tertile of Mediterranean diet score). Multiple linear regression analyses, controlled for potential confounders, revealed that an increment in soft drinks consumption of $100 \mathrm{ml}$ was associated with a $0 \cdot 21 \mathrm{~kg} / \mathrm{m}^{2}$ increase in BMI $(P=0 \cdot 001)$. Only implausibly low reports of energy consumption showed a null association between soft drinks consumption and BMI.

Conclusions: Soft drinks consumption was not embedded in a healthy diet context and was positively associated with BMI and sedentary lifestyle in this Mediterranean population.
\end{abstract}

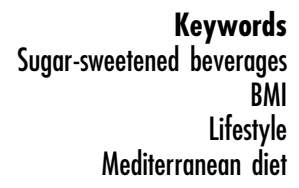

The obesity epidemic is one of the most important challenges for public health policy. The prevalence of excessive weight strongly affects cardiovascular health ${ }^{(1,2)}$ and contributes to a tremendous economic burden of public health $^{(3)}$. An increasing trend of obesity has been observed during the last decade not only in the USA but also in Europe, and particularly in Spain ${ }^{(4)}$. Between 1995 and 2000, BMI increased by about $1 \mathrm{~kg} / \mathrm{m}^{2}$ in men and women at the population level in north-east Spain ${ }^{(5)}$.
The increase in excessive weight is accompanied by concomitant lifestyle changes, such as in diet and physical activity $^{(6)}$. Total energy intake has increased during the past 20 years in the USA, a trend driven mainly by an increase in carbohydrate consumption ${ }^{(7)}$. The daily energy contribution from soft drinks increased from $1000 \mathrm{~kJ}(239 \mathrm{kcal})$ to $1230 \mathrm{~kJ}(294 \mathrm{kcal})$ over the past decade among adult North American soft drinks consumers $^{(8,9)}$. In Spain, daily soft drinks consumption has 
also increased, albeit slightly, from $44.8 \mathrm{ml}(79 \mathrm{~kJ} /$ $18.8 \mathrm{kcal})$ in 1991 to $72 \cdot 4 \mathrm{ml}(127 \mathrm{~kJ} / 30 \cdot 4 \mathrm{kcal})$ in $2001^{(10)}$.

Although still inconclusive, the main body of evidence associating soft drinks consumption with BMI comes from American studies focusing on children and adolescents $^{(11-14)}$. Our knowledge of the impact of soft drinks consumption on BMI in the adult population is limited ${ }^{(15)}$, particularly where the consumption is low, as in Mediterranean populations. Lifestyle differs by population and culture, and therefore the underlying dietary pattern and leisure-time physical activity involving soft drinks might vary and might have different impacts on the association of soft drinks consumption with BMI. Indeed, one might speculate that soft drinks consumption might not produce detrimental health outcomes within the context of a highquality diet such as the Mediterranean diet. Furthermore, energy misreporting is a common problem in nutritional studies and, if not controlled for, results in biased findings about the impact of diet on $\mathrm{BMI}^{(16,17)}$.

The aim of the present study was to analyse the association of soft drinks consumption with BMI and the risk of obesity, controlling for lifestyle and low energy reporting, in a representative Mediterranean population.

\section{Materials and methods}

\section{Study participants}

Data were obtained from population-based cross-sectional surveys conducted in Girona (Spain) in 2000 and 2005. These surveys of randomly selected, free-living men and women included 3058 persons aged 25-74 years in 2000 and 6352 persons aged 35-80 years in 2005. Response rates for the two surveys were $71.0 \%$ and $71.5 \%$, respectively. All survey participants aged 35 to 74 years ( $n$ 8195) were included in the present study. After excluding persons with extremely low reported energy intake $(<3347 \mathrm{~kJ} /<800 \mathrm{kcal})$ and extremely high value for BMI $\left(>60 \mathrm{~kg} / \mathrm{m}^{2}\right)$ or reported energy consumption (corresponding to a physical activity level $>2 \cdot 4$, which exceeds the established upper limit $^{(18)}$ for strenuous work or highly active leisure behaviours), 3593 men and 3508 women were included for analysis.

The protocol was approved by the local ethics committee (CEIC-IMAS, Barcelona, Spain) and all participants were sent the results of their examination.

\section{Antbropometric measurements}

A precision scale was used for weight measurement, with subjects in underwear. Readings were rounded to the nearest $200 \mathrm{~g}$. Height was measured in the standing position and measurements rounded to the nearest $5 \mathrm{~mm}$. BMI was determined as weight divided by height squared $\left(\mathrm{kg} / \mathrm{m}^{2}\right)$.

\section{Dietary assessment}

Standard structured questionnaires administered by trained personnel were used to obtain information on demographic and socio-economic variables, medical history, diet and lifestyle factors, including tobacco smoking and alcohol consumption. Food consumption and nutrient intake were measured by a 168 -item validated $\mathrm{FFQ}^{(19)}$ administered by a trained interviewer. The optical readable FFQ asked for usual intake over the past year of specific foods and alcoholic and non-alcoholic beverages. Participants were asked to describe their average frequency of consuming each item, using ten categories ranging from 'almost never' to ' 6 times/ day'. Instead of standard questions on portion size based on weight or volume, the FFQ used specific medium servings, defined by natural (e.g. one orange, one slice of bread) or household units (e.g. one spoon, one cup, one glass). The FFQ included one item on soft drinks consumption (' 1 can of sugar-sweetened carbonated soda: Coca Cola, Fanta or similar, but not light'). Therefore, in the current study the term 'soft drinks consumption' is limited to sugar-sweetened carbonated beverages.

\section{Measurement of diet quality}

Overall diet quality was measured as adherence to the Mediterranean dietary pattern, using the Mediterranean diet score (MDS). Higher scores indicate higher adherence to the Mediterranean diet. Distribution values were calculated for all dietary components of the FFQ. The resulting MDS ranged from 10 to 30 .

This operative variable for the analysis of associations between diet quality and health outcomes is calculated according to the tertile distribution of food consumption, with the exception of red wine. For cereals, fruits, vegetables, legumes, fish, olive oil and nuts, the lowest tertile is coded as 1 , medium as 2 and the highest as 3 . The score is inverted for meat and dairy products, with the highest tertile coded as 1 and the lowest as 3. Moderate red wine consumption (up to $20 \mathrm{~g}$ ) is included as a favourable component in the MDS, with a score of 3. Exceeding this upper limit or reporting no red wine consumption was coded as 0 .

BMR was calculated using the predictive equations based on sex, age and body weight recommended by $\mathrm{FAO} / \mathrm{WHO} /$ United Nations University ${ }^{(20)}$. If the quotient of reported energy intake divided by the predicted BMR was $<1 \cdot 2$, this was considered as energy under-reporting.

\section{Other measurements}

Leisure-time physical activity was measured by the Minnesota Leisure-Time Physical Activity Questionnaire and administered by a trained interviewer. This questionnaire has been previously validated for Spanish men and women ${ }^{(21,22)}$. Sedentary lifestyle was defined as leisure-time physical activity of less than $30 \mathrm{~min} / \mathrm{d}$.

Information on smoking habits was obtained by structured interview. Participants were categorized as non-smokers or current smokers.

Maximum education level attained was elicited and recorded for analysis as primary school, secondary school and post-secondary school. 
Table 1 General characteristics of the study population according to frequency of soft drinks consumption*: random sample of the 35- to 74-year-old population (3910 men and 4285 women), Girona, Spain

\begin{tabular}{|c|c|c|c|c|c|}
\hline & \multicolumn{2}{|c|}{$\begin{array}{l}\text { Soft drinks non-consumers } \\
\qquad(n \text { 4141) }\end{array}$} & \multicolumn{2}{|c|}{$\begin{array}{l}\text { Soft drinks consumers } \\
(n \text { 2960) }\end{array}$} & \multirow[b]{2}{*}{$P$} \\
\hline & $\%$ or Mean & $95 \% \mathrm{Cl}$ or SD & $\%$ or Mean & $95 \% \mathrm{Cl}$ or SD & \\
\hline Women (\%) & $52 \cdot 2$ & $51 \cdot 7,54 \cdot 7$ & $44 \cdot 1$ & $42 \cdot 3,45 \cdot 9$ & $<0.001$ \\
\hline Age (years) & $57 \cdot 1$ & $10 \cdot 3$ & $50 \cdot 4$ & $10 \cdot 5$ & $<0.001$ \\
\hline Total energy intake (MJ/d) & $9 \cdot 6$ & $2 \cdot 6$ & $10 \cdot 5$ & $2 \cdot 7$ & $<0.001$ \\
\hline Low energy reporterst (\%) & $23 \cdot 3$ & $22 \cdot 2,24 \cdot 5$ & $16 \cdot 2$ & $14 \cdot 9,17 \cdot 6$ & $<0.001$ \\
\hline Dietary energy density $\ddagger$ & $1 \cdot 23$ & 0.32 & $1 \cdot 38$ & 0.32 & $<0.001$ \\
\hline BMI $\left(\mathrm{kg} / \mathrm{m}^{2}\right)$ & $27 \cdot 8$ & $4 \cdot 5$ & $27 \cdot 3$ & $4 \cdot 5$ & 0.009 \\
\hline LTPA $($ MET $\times \min / \mathrm{d})$ & $307 \cdot 9$ & $324 \cdot 7$ & $296 \cdot 9$ & 311.9 & 0.031 \\
\hline Smokers (\%) & 20.5 & $19 \cdot 2,21 \cdot 8$ & $28 \cdot 2$ & $26 \cdot 6,29 \cdot 7$ & $<0.001$ \\
\hline Alcohol consumption (g/d) & $11 \cdot 5$ & $17 \cdot 2$ & $12 \cdot 1$ & $19 \cdot 8$ & 0.369 \\
\hline Educational level§ (\%) & $41 \cdot 3$ & $39 \cdot 8,42 \cdot 8$ & $53 \cdot 6$ & $51 \cdot 8,55 \cdot 3$ & $<0.001$ \\
\hline
\end{tabular}

LTPA, leisure-time physical activity; MET, metabolic equivalent task.

${ }^{*}$ Results are expressed as percentage of subjects and $95 \%$ confidence interval or mean and standard deviation. Significance of $P$ between soft drinks consumers and soft drinks non-consumers was determined by the Student $t$ test (continuous variables) or logistical regression analysis (categorical variables). tEnergy intake:BMR $<1 \cdot 2$.

‡Energy density was calculated as energy intake from all foods consumed (kcal) divided by weight of foods consumed $(\mathrm{g})$; $1 \mathrm{kcal}=4 \cdot 184 \mathrm{~kJ}$.

\$More than primary school.

Energy density was defined as the amount of energy $(\mathrm{kJ})$ in a given weight of food $(\mathrm{g})$.

\section{Statistical analysis}

Differences in continuous variables were compared using the Student $t$ test. The $\chi^{2}$ test was used for categorical variables. General linear modelling procedures (PROC GLM) in the SAS statistical software package version $9 \cdot 1$ (SAS Institute Inc., Cary, NC, USA) were used to estimate lifestyle, anthropometric and socio-economic variables according to categories of soft drinks consumption. For continuous variables, polynomial contrast was calculated to determine $P$ for linear trend.

Multiple linear regression models were fitted (PROC REG procedure in SAS version 9.1) to determine the confounder-controlled association of soft drinks consumption and BMI, and produced a normal distribution of data. Multiple linear regression analysis, stratified by energy misreporting, was performed to determine the impact of energy under-reporting on the association between soft drinks consumption and BMI.

Multiple logistic regression analysis (PROC LOGISTIC procedure in SAS version 9.1) was used to assess the relationship of daily soft drinks consumption $(200 \mathrm{ml} / \mathrm{d})$ and diet quality (tertile distribution of the MDS). Differences were considered significant if $P<0 \cdot 05$.

\section{Results}

Less than half of the population ( $41.7 \%)$ consumed soft drinks. Mean daily consumption of soft drinks consumers was $86.2 \mathrm{ml}$; monthly, weekly and daily frequency of soft drinks consumption was $55 \cdot 5 \%(19 \cdot 0 \mathrm{ml} / \mathrm{d}), 27 \cdot 7 \%$ $(77 \cdot 9 \mathrm{ml} / \mathrm{d})$ and $16 \cdot 8 \%(326 \cdot 4 \mathrm{ml} / \mathrm{d})$, respectively.
The association of soft drinks consumption with BMI, obesity and lifestyle was quite similar for both sexes. For this reason we present non-stratified results, adjusted for sex as appropriate.

Soft drinks consumers were younger, more highly educated and less prone to under-report total energy intake. They reported higher energy intakes, spent less time in leisure-time physical activity, smoked more and had a lower BMI than non-consumers (Table 1). Age decreased across frequencies of soft drinks consumption (Table 2). After controlling for sex and age, higher frequency of soft drinks consumption was associated with higher BMI and higher prevalence of sedentary lifestyle and obesity (Table 2).

Soft drinks consumption was directly associated with energy intake, energy density and intakes of carbohydrates, pastry/sweets and high-fat dairy products (Table 3). A decrease occurred across categories of soft drinks consumption in the ratio of unsaturated to saturated fat and intakes of protein, total fat, fibre, olive oil, low-fat dairy products, fish, vegetables, fruits, nuts, poultry/rabbit and legumes (Table 3).

Multiple linear regression analysis - adjusted for sex, age, educational status, leisure-time physical activity, energy intake, smoking, alcohol consumption and energy under-reporting - revealed that a $100 \mathrm{ml}$ increment in soft drinks consumption was associated with an increase of $0 \cdot 213 \mathrm{~kg} / \mathrm{m}^{2}$ in BMI $(P<0 \cdot 001$; Table 4$)$. This association was unchanged in both magnitude and direction in plausible energy reporters but was attenuated in low energy reporters. Multivariate ANOVA adjusted for sex and age revealed a positive association of soft drinks consumption across BMI categories (normal weight, overweight and obese; $P<0 \cdot 001)$. Participants with normal weight consumed $31 \cdot 2 \mathrm{ml} / \mathrm{d}$ whereas their obese 
Table 2 General characteristics of the population by frequency of soft drinks consumption*: random sample of the 35- to 74-year-old population (3910 men and 4285 women), Girona, Spain

\begin{tabular}{|c|c|c|c|c|c|c|c|c|c|}
\hline & \multicolumn{2}{|c|}{ No consumption ( $n$ 4141) } & \multicolumn{2}{|c|}{ Monthly ( $n$ 1644) } & \multicolumn{2}{|c|}{ Weekly ( $n$ 820) } & \multicolumn{2}{|c|}{ Daily (n 496) } & \multirow{2}{*}{$\begin{array}{c}P \text { for } \\
\text { linear treno }\end{array}$} \\
\hline & $\%$ or Mean & $95 \% \mathrm{Cl}$ & $\%$ or Mean & $95 \% \mathrm{Cl}$ & $\%$ or Mean & $95 \% \mathrm{Cl}$ & $\%$ or Mean & $95 \% \mathrm{Cl}$ & \\
\hline Sex (\% men) & $46 \cdot 8$ & $45 \cdot 3,48 \cdot 3$ & $49 \cdot 3$ & $46 \cdot 9,51 \cdot 7$ & $61 \cdot 2$ & $57 \cdot 8,64 \cdot 6$ & $69 \cdot 0$ & $64 \cdot 6,73 \cdot 3$ & $<0.001$ \\
\hline Age (years) & $57 \cdot 1$ & $56 \cdot 8,57 \cdot 4$ & $51 \cdot 0$ & $50 \cdot 5,51 \cdot 5$ & $49 \cdot 0$ & $48 \cdot 3,49 \cdot 7$ & $50 \cdot 1$ & $49 \cdot 2,51 \cdot 0$ & $<0.001$ \\
\hline BMl $\left(\mathrm{kg} / \mathrm{m}^{2}\right)$ & $27 \cdot 5$ & $27 \cdot 4,27 \cdot 7$ & $27 \cdot 6$ & $27 \cdot 4,27 \cdot 8$ & $28 \cdot 2$ & $27 \cdot 8,28 \cdot 5$ & $28 \cdot 1$ & $27 \cdot 7,28 \cdot 5$ & 0.001 \\
\hline Obesityt (\%) & $25 \cdot 6$ & $24 \cdot 3,27 \cdot 0$ & $26 \cdot 7$ & $24 \cdot 5,28 \cdot 8$ & $29 \cdot 3$ & $26 \cdot 2,32 \cdot 3$ & $30 \cdot 2$ & $26 \cdot 3,34 \cdot 1$ & 0.007 \\
\hline Overweightł (\%) & $44 \cdot 1$ & $42 \cdot 6,45 \cdot 7$ & $44 \cdot 1$ & $41 \cdot 7,48 \cdot 5$ & $45 \cdot 1$ & $41 \cdot 7,48 \cdot 5$ & $45 \cdot 3$ & $40 \cdot 9,49 \cdot 6$ & 0.551 \\
\hline LTPA $(M E T \times \mathrm{min} / \mathrm{d})$ & 303 & 293,313 & 309 & 294, 324 & 303 & 281,325 & 285 & 257,313 & $0 \cdot 280$ \\
\hline Sedentary lifestyle§ (\%) & $32 \cdot 4$ & $31 \cdot 0,33 \cdot 9$ & $30 \cdot 2$ & $27 \cdot 9,32 \cdot 5$ & $33 \cdot 4$ & $30 \cdot 2,36 \cdot 7$ & $39 \cdot 5$ & $35 \cdot 3,43 \cdot 6$ & 0.025 \\
\hline Smokers (\%) & $23 \cdot 7$ & $22 \cdot 4,25 \cdot 0$ & $21 \cdot 8$ & $19 \cdot 8,23 \cdot 8$ & $23 \cdot 3$ & $20 \cdot 5,26 \cdot 1$ & $30 \cdot 5$ & $26 \cdot 8,34 \cdot 1$ & $0 \cdot 175$ \\
\hline Alcohol consumption (g) & $10 \cdot 4$ & $10 \cdot 0,10 \cdot 9$ & $9 \cdot 8$ & $9 \cdot 1 \cdot 10 \cdot 5$ & $8 \cdot 7$ & $7 \cdot 7,9 \cdot 7$ & $9 \cdot 6$ & $8 \cdot 4,10 \cdot 9$ & 0.093 \\
\hline Educational levelll (\%) & $45 \cdot 4$ & $44 \cdot 0,46 \cdot 9$ & $52 \cdot 3$ & $50 \cdot 0,54 \cdot 6$ & $44 \cdot 6$ & $41 \cdot 3,47 \cdot 9$ & $37 \cdot 9$ & $33 \cdot 7,42 \cdot 1$ & 0.075 \\
\hline
\end{tabular}

LTPA, leisure-time physical activity; MET, metabolic equivalent task.

${ }^{\star}$ Results are expressed as percentage of subjects or mean and $95 \%$ confidence interval. Sex- and age-adjusted ANOVA was used to estimate variables according to frequency of soft drinks consumption.

$+\mathrm{BMI} \geq 30 \mathrm{~kg} / \mathrm{m}^{2}$.

$\neq B M I=25 \cdot 0-29 \cdot 9 \mathrm{~kg} / \mathrm{m}^{2}$.

$\S$ Less than $30 \mathrm{~min}$ LTPA $/ \mathrm{d}$.

IIMore than primary school.

Table 3 Age- and sex-adjusted daily food and nutrient intakes by category of soft drinks consumption*: random sample of the 35- to 74year-old population (3910 men and 4285 women), Girona, Spain

\begin{tabular}{|c|c|c|c|c|c|c|c|c|c|}
\hline & \multicolumn{2}{|c|}{$\begin{array}{l}\text { No consumption } \\
(0 \mathrm{ml} ; n 4141)\end{array}$} & \multicolumn{2}{|c|}{$\begin{array}{c}\text { Monthly } \\
(19.0 \mathrm{ml} ; n \text { 1644) }\end{array}$} & \multicolumn{2}{|c|}{$\begin{array}{c}\text { Weekly } \\
(77.9 \mathrm{ml} ; n \text { 820) }\end{array}$} & \multicolumn{2}{|c|}{$\begin{array}{c}\text { Daily } \\
\text { (326.4 ml; } n \text { 496) }\end{array}$} & \multirow{2}{*}{$\begin{array}{c}P \text { for } \\
\text { linear trend }\end{array}$} \\
\hline & Mean or $\%$ & $95 \% \mathrm{Cl}$ & Mean or $\%$ & $95 \% \mathrm{Cl}$ & Mean or $\%$ & $95 \% \mathrm{Cl}$ & Mean or $\%$ & $95 \% \mathrm{Cl}$ & \\
\hline Energy (MJ) & $9 \cdot 7$ & $9 \cdot 6,9 \cdot 8$ & $10 \cdot 1$ & $9 \cdot 9,10 \cdot 2$ & $10 \cdot 4$ & $10 \cdot 2,10 \cdot 6$ & $10 \cdot 9$ & $10 \cdot 7,11 \cdot 1$ & $<0.001$ \\
\hline Energy from soft drinks (MJ) & 0 & - & 0.03 & $0.02,0.04$ & 0.13 & $0 \cdot 12,0 \cdot 14$ & 0.56 & $0.55,0.57$ & $<0.001$ \\
\hline Energy density $\dagger$ & $1 \cdot 25$ & $1 \cdot 24,1 \cdot 26$ & $1 \cdot 32$ & $1 \cdot 30,1 \cdot 33$ & $1 \cdot 36$ & $1 \cdot 33,1 \cdot 38$ & $1 \cdot 39$ & $1 \cdot 36,1 \cdot 42$ & $<0.001$ \\
\hline Energy under-reporting‡ (\%) & $23 \cdot 9$ & $22 \cdot 6,25 \cdot 1$ & $19 \cdot 1$ & $17 \cdot 1,21 \cdot 0$ & $17 \cdot 5$ & $14 \cdot 7,20 \cdot 3$ & $14 \cdot 2$ & $10 \cdot 6,17 \cdot 8$ & $<0.001$ \\
\hline Carbohydrate $(\%)$ & $40 \cdot 9$ & $40 \cdot 7,41 \cdot 2$ & $40 \cdot 5$ & $40 \cdot 2,40 \cdot 9$ & $41 \cdot 9$ & $41 \cdot 4,42 \cdot 4$ & $44 \cdot \overline{4}$ & $43 \cdot 8,45 \cdot 1$ & $<0.001$ \\
\hline Protein§ (\%) & $17 \cdot 9$ & $17 \cdot 9,18 \cdot 0$ & $17 \cdot 6$ & $17 \cdot 4,17 \cdot 7$ & $17 \cdot 3$ & $17 \cdot 1,17 \cdot 4$ & $16 \cdot 4$ & $16 \cdot 2,16 \cdot 7$ & $<0.001$ \\
\hline Fat $(\%)$ & $40 \cdot 8$ & $40 \cdot 7,41 \cdot 1$ & $41 \cdot 8$ & $41 \cdot 5,42 \cdot 2$ & $41 \cdot 2$ & $40 \cdot 7,41 \cdot 6$ & 39.9 & $39 \cdot 1,40 \cdot 3$ & $<0.001$ \\
\hline Unsaturated:saturated fat & $2 \cdot 30$ & $2 \cdot 28,2 \cdot 31$ & $2 \cdot 22$ & $2 \cdot 19,2 \cdot 25$ & $2 \cdot 14$ & $2 \cdot 11,2 \cdot 19$ & $2 \cdot 18$ & $2 \cdot 14,2 \cdot 24$ & $<0.001$ \\
\hline Dietary fibre (g/4·18 MJ) & $12 \cdot 3$ & $12 \cdot 1,12 \cdot 4$ & $11 \cdot 3$ & $11 \cdot 1,11 \cdot 5$ & $10 \cdot 7$ & $10 \cdot 4,11 \cdot 0$ & $10 \cdot 2$ & $9 \cdot 9,10 \cdot 6$ & $<0.002$ \\
\hline Vegetables $(\mathrm{g} / 4 \cdot 18 \mathrm{MJ})$ & 226 & 222,230 & 204 & 198,210 & 191 & 182,200 & 181 & 171,192 & $<0.001$ \\
\hline Fruits (g/4.18MJ) & 207 & 203,212 & 184 & 178, 192 & 176 & 166,186 & 164 & 151,176 & $<0.001$ \\
\hline Legumes $(\mathrm{g} / 4 \cdot 18 \mathrm{MJ})$ & $23 \cdot 7$ & $23 \cdot 2,24 \cdot 2$ & $21 \cdot 8$ & $21 \cdot 0,22 \cdot 6$ & $21 \cdot 6$ & $20 \cdot 5,22 \cdot 8$ & $21 \cdot 4$ & $20 \cdot 0,22 \cdot 9$ & 0.005 \\
\hline Cereals $(\mathrm{g} / 4 \cdot 18 \mathrm{MJ})$ & $75 \cdot 2$ & $74 \cdot 2,73 \cdot 6$ & $75 \cdot 0$ & $73 \cdot 4,76 \cdot 6$ & $75 \cdot 9$ & $73 \cdot 6,78 \cdot 1$ & $74 \cdot 1$ & $71 \cdot 1,76 \cdot 9$ & 0.568 \\
\hline$\cdot 18 \mathrm{MJ})$ & $35 \cdot 5$ & $34 \cdot 9,36 \cdot 1$ & $33 \cdot 9$ & $32 \cdot 9,34 \cdot 9$ & $32 \cdot 1$ & $30 \cdot 7,33 \cdot 5$ & $29 \cdot 8$ & $28 \cdot 0,31 \cdot 6$ & $<0.001$ \\
\hline Red meat/sausages (g/4.18 MJ) & $45 \cdot 9$ & $45 \cdot 3,46 \cdot 6$ & $48 \cdot 7$ & $47 \cdot 7,49 \cdot 9$ & $49 \cdot 1$ & $47 \cdot 6,50 \cdot 7$ & $47 \cdot 4$ & $45 \cdot 4,49 \cdot 3$ & $0 \cdot 167$ \\
\hline Poultry/rabbit $(\mathrm{g} / 4 \cdot 18 \mathrm{MJ})$ & $19 \cdot 5$ & $19 \cdot 1,20 \cdot 0$ & $18 \cdot 2$ & $17 \cdot 4,18 \cdot 9$ & $17 \cdot 6$ & $16 \cdot 6,18 \cdot 7$ & $16 \cdot 9$ & $15 \cdot 5,18 \cdot 2$ & $<0.001$ \\
\hline Olive oil $(\mathrm{g} / 4 \cdot 18 \mathrm{MJ})$ & $11 \cdot 8$ & $11 \cdot 6,12 \cdot 0$ & $11 \cdot \overline{6}$ & $11 \cdot 2,12 \cdot 0$ & $10 \cdot 5$ & $10 \cdot 0,11 \cdot 0$ & $10 \cdot 3$ & $9 \cdot 7,11 \cdot 0$ & $<0.001$ \\
\hline Low-fat dairy $(\mathrm{g} / 4 \cdot 18 \mathrm{MJ})$ & $78 \cdot 3$ & $75 \cdot 6,81 \cdot 0$ & $67 \cdot 3$ & $63 \cdot 1,71 \cdot 5$ & $61 \cdot 7$ & $55 \cdot 8,67 \cdot 7$ & $51 \cdot 4$ & $43 \cdot 8,59 \cdot 1$ & $<0.001$ \\
\hline High-fat dairy (g/4·18 MJ) & $57 \cdot 2$ & $55 \cdot 1,59 \cdot 4$ & $59 \cdot 4$ & $56 \cdot 1,62 \cdot 8$ & $66 \cdot 8$ & $62 \cdot 1,71 \cdot 6$ & $64 \cdot 5$ & $58 \cdot 2,70 \cdot 5$ & 0.006 \\
\hline ets $(g / 4 \cdot 18 \mathrm{MJ})$ & $22 \cdot 1$ & $21 \cdot 5,22 \cdot 8$ & $25 \cdot 6$ & $24 \cdot 5,26 \cdot 6$ & $29 \cdot 1$ & $27 \cdot 6,30 \cdot 5$ & $30 \cdot 1$ & $28 \cdot 3,31 \cdot 9$ & $<0.001$ \\
\hline Nuts $(\mathrm{g} / 4 \cdot 18 \mathrm{MJ})$ & $6 \cdot 4$ & $6 \cdot 2,6 \cdot 7$ & $6 \cdot 0$ & $5 \cdot 6,6 \cdot 4$ & $5 \cdot 2$ & $4 \cdot 7,5 \cdot 8$ & $4 \cdot 4$ & $3 \cdot 7,5 \cdot 1$ & $<0.001$ \\
\hline
\end{tabular}

${ }^{*}$ Results are expressed as mean or percentage of subjects and 95\% confidence interval. Sex- and age-adjusted ANOVA was used to estimate variables according to frequency of soft drinks consumption.

tEnergy density was calculated as energy intake from all foods consumed (kcal) divided by weight of foods consumed $(\mathrm{g}) ; 1 \mathrm{kcal}=4 \cdot 184 \mathrm{~kJ}$.

‡Energy intake:BMR $<1 \cdot 2$.

$\S$ Percentage of total energy intake.

peers drank $42 \cdot 4 \mathrm{ml} / \mathrm{d}(P=0 \cdot 003)$. Additionally controlling for energy intake, leisure-time physical activity, smoking, educational level, diet quality and energy under-reporting attenuated this association slightly $(P$ for linear trend $=$ $0 \cdot 007$; soft drinks consumption by normal weight $v$. obese participants: $32 \cdot 2 \mathrm{ml} / \mathrm{d} v .41 \cdot 5 \mathrm{ml} / \mathrm{d} ; P=0 \cdot 021$ ). Figure 1 shows the association of soft drinks consumption with overall diet quality, defined by MDS. Adherence to a healthy diet decreased with daily soft drinks consumption $(P<0 \cdot 001)$.

\section{Discussion}

Soft drinks consumption was directly associated with BMI. Stratifying by implausibly low and plausible energy reporters revealed a significant association of soft drinks consumption and BMI in plausible reporters but not in under-reporters. Furthermore, daily soft drinks consumption was associated with low diet quality.

Soft drinks consumption in the USA constitutes $7 \cdot 1 \%$ of total energy intake ${ }^{(23)}$. In contrast, in the present population 
Table 4 Regression coefficient and $95 \%$ confidence interval of the association between soft drinks consumption and $\mathrm{BMI}^{\star}$ among a random sample of the 35- to 74-year-old population (3910 men and 4285 women), Girona, Spain

\begin{tabular}{|c|c|c|c|}
\hline & \multicolumn{3}{|c|}{ BMI $\left(\mathrm{kg} / \mathrm{m}^{2}\right)$} \\
\hline & Regression coefficient & $95 \% \mathrm{Cl}$ & $P$ \\
\hline \multicolumn{4}{|l|}{ All participants $(n$ 7101)† } \\
\hline Soft drinks $(100 \mathrm{ml}) \ddagger$ & $0 \cdot 213$ & $0 \cdot 112,0 \cdot 313$ & $<0.001$ \\
\hline \multicolumn{4}{|l|}{ Plausible energy reporter $(n 5585) \S$} \\
\hline Soft drinks $(100 \mathrm{ml}) \ddagger$ & $0 \cdot 218$ & $0 \cdot 114,0 \cdot 322$ & $<0.001$ \\
\hline \multicolumn{4}{|l|}{ Low energy reporter $(n$ 1512)§ } \\
\hline Soft drinks (100 ml) & $0 \cdot 185$ & $-0.142,0.512$ & 0.267 \\
\hline
\end{tabular}

*Multiple linear regression models were fitted to analyse the association of soft drinks consumption and BMI.

tAdjusted for sex, age, leisure-time physical activity, educational level, smoking, alcohol consumption, diet quality (Mediterranean diet score), energy (energy from soft drinks excluded) and energy under-reporting (energy intake:BMR $<1 \cdot 2$ ).

$\$ 100 \mathrm{ml}$ corresponds to 0.5 drinks/d.

$\S$ Adjusted for sex, age, leisure-time physical activity, educational level, smoking, alcohol consumption, diet quality (Mediterranean diet score) and energy (energy from soft drinks excluded).

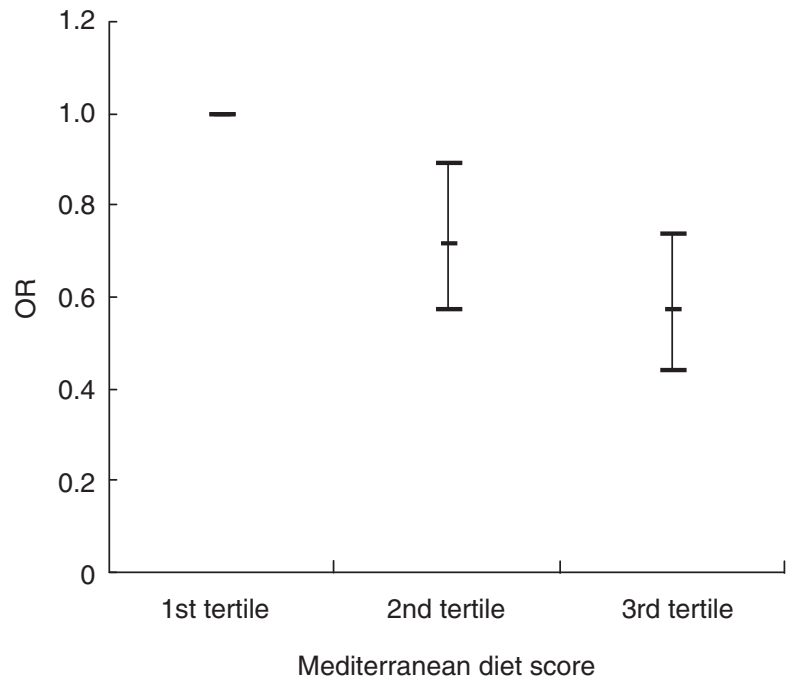

Fig. 1 Odds ratio and $95 \%$ confidence interval of daily soft drinks consumption and adherence to the Mediterranean diet (tertile distribution of the Mediterranean diet score) among a random sample of the 35- to 74-year-old population (3910 men and 4285 women), Girona, Spain. Odds ratios were adjusted for sex, age, leisure-time physical activity, educational level, smoking, alcohol consumption, diet quality (Mediterranean diet score), energy (energy from soft drinks excluded) and energy under-reporting (energy intake:BMR <1.2). $P$ for linear trend $=0.001$

soft drinks consumption can be considered low, since it contributes only $61 \mathrm{~kJ}(14.5 \mathrm{kcal})$ to total energy intake ( $0.6 \%$ of the total energy supply). Furthermore, the average daily amount $(86.8 \mathrm{ml})$ reported by soft drinks consumers falls within the range of the recently proposed tolerable intake of soft drinks ${ }^{(24)}$. The Mediterranean dietary pattern is associated with favourable health outcomes ${ }^{(25-27)}$. It is conceivable that soft drinks consumption at these amounts is unlikely to replace healthy food choices in this context. Furthermore, moderate soft drinks consumption as part of a healthy lifestyle should not affect weight gain, cardiovascular health or BMI. However, our data showed that frequent soft drinks consumption increased the risk of low adherence to the Mediterranean diet and was associated with a cluster of unhealthy food preferences. The observation that unhealthy food choices are associated with soft drinks consumption is in line with previously published reports from younger populations ${ }^{(28,29)}$.

In the present population there was a positive association, controlled for potential confounders, between soft drinks consumption and BMI. Limited evidence from other cross-sectional and prospective epidemiological studies in adult populations, particularly from the USA, has indicated a modest positive association ${ }^{(11-15)}$. Chen et al. reported a significant weight loss associated with a reduction of 1 serving of soft drinks per day after 18 months ${ }^{(15)}$. A recently published Spanish study of male and female university alumni found a significant positive association between soft drinks consumption and weight gain among adults who had gained more than $3 \mathrm{~kg}$ weight in the 5 years before the study ${ }^{(30)}$. In the present study, a $100 \mathrm{ml}$ increment in soft drinks consumption was associated with an increase of $0 \cdot 21 \mathrm{~kg} / \mathrm{m}^{2}$ in BMI. Although the magnitude of the association is modest, the fact that it derives from a single food item, rather than a complex dietary pattern, reflects a considerable impact on BMI in this population. The association of BMI and soft drinks consumption was only slightly attenuated after controlling for overall diet quality. This finding indicates that soft drinks consumption affects BMI independently of the magnitude of diet quality as measured by adherence to the Mediterranean diet. Even within the context of an overall healthy diet, soft drinks consumption adversely affects BMI.

Several mechanisms linking soft drinks consumption and weight gain have been proposed ${ }^{(31)}$. The predominant evidence points to an imbalance in energy intake, as soft drinks mediate less satiation and low satiety $^{(32)}$. In the present population, higher soft drinks consumption was not associated with energy compensation in the overall diet. By definition, excess energy intake through soft drinks consumption without compensatory energy expenditure is a formula for weight gain in the long run. 
Unfortunately, energy under-reporting is a common problem for the analysis of associations between diet and health outcomes, particularly $\mathrm{BMI}^{(16,17)}$. It has been shown that obese subjects tend to selectively under- or overestimate selected foods ${ }^{(16,17)}$. In the present study, prevalence of low energy reporting decreased with frequent soft drinks consumption. Thus, it was not surprising that soft drinks consumption was positively associated with BMI in plausible reporters but exhibited a null association in those who reported low energy consumption. Our results indicate that controlling for energy under-reporting is essential to avoid bias in the association of foods or diet and BMI.

A limitation of the present study's cross-sectional design is that causality cannot be drawn between the variables studied. Misreporting is an acknowledged source of measurement error in prospective or retrospective methods of dietary assessment using self-reported food intake records. Furthermore, total consumption of sugar-sweetened drinks is underestimated in this population because the term 'soft drink' did not include non-carbonated sugar-sweetened beverages. The strengths of the present study include the relatively large sample size, the population-based design and the consideration of potential confounders in the analysis, including physical activity, smoking, socio-economic status and energy under-reporting.

In conclusion, frequent consumers of soft drinks tended towards a more sedentary lifestyle and a cluster of unhealthy dietary habits. The risk of low adherence to the Mediterranean diet increased significantly with daily soft drinks consumption. Higher BMI was found in more frequent consumers of soft drinks as compared with their nonconsuming peers, after controlling for several potential confounders. Low energy reporters showed a null association between soft drinks consumption and BMI, indicating that energy misreporting is a strong potential confounder for analysis of the relationship between soft drinks consumption and BMI.

\section{Acknowledgements}

This work was supported by a grant from the Instituto de Salud Carlos III FEDER (PI080439) and a joint contract of the Instituto de Salud Carlos III and the Health Department of the Catalan Government (Generalitat de Catalunya), CP 03/00115. The CIBEROBN is an initiative from the Instituto de Salud Carlos III, Madrid, Spain. The authors declare no conflict of interest. E.B. and M.D.-N. contributed equally to the study, and each can be regarded as first author of the present paper. The manuscript was prepared by E.B. and M.D.-N., with significant input and feedback from all co-authors. H.S. contributed to the discussion and interpretation of results and writing of the manuscript. R.P.-L., T.R.-G., A.R., G.G.-Z., J.M.-O., S.V.-H. and M.P.-C. provided expertise in data analysis and in interpretation and discussion of the results. They made substantial suggestions on the manuscript. The authors appreciate the English revision by Elaine M. Lilly, $\mathrm{PhD}$ (Writers First Aid).

\section{References}

1. Rashid MN, Fuentes F, Touchon RC et al. (2003) Obesity and the risk for cardiovascular disease. Prev Cardiol 6, 42-47.

2. Poirier P, Giles TD, Bray GA et al. (2006) Obesity and cardiovascular disease: pathophysiology, evaluation, and effect of weight loss: an update of the 1997 American Heart Association Scientific Statement on Obesity and Heart Disease from the Obesity Committee of the Council on Nutrition, Physical Activity, and Metabolism. Circulation 113, 898-918.

3. Müller-Riemenschneider F, Reinhold T, Berghöfer A et al. (2008) Health-economic burden of obesity in Europe. Eur J Epidemiol 23, 499-509.

4. García-Alvarez A, Serra-Majem L, Ribas-Barba L et al. (2007) Obesity and overweight trends in Catalonia, Spain (1992-2003): gender and socio-economic determinants. Public Health Nutr 10, 1368-1378.

5. Schröder H, Elosua R, Vila J et al. (2007) Secular trends of obesity and cardiovascular risk factors in a Mediterranean population. Obesity (Silver Spring) 15, 557-562.

6. Nooyens AC, Visscher TL, Schuit AJ et al. (2005) Effects of retirement on lifestyle in relation to changes in weight and waist circumference in Dutch men: a prospective study. Public Health Nutr 8, 1266-1274.

7. Briefel RR \& Johnson CL (2004) Secular trends in dietary intake in the United States. Annu Rev Nutr 24, 401-431.

8. Nielsen SJ \& Popkin BM (2004) Changes in beverage intake between 1977 and 2001. Am J Prev Med 27, 205-210.

9. Bleich SN, Wang YC, Wang Y et al. (2009) Increasing consumption of sugar-sweetened beverages among US adults: 1988-1994 to 1999-2004. Am J Clin Nutr 89, 372-381.

10. Asociación Española de Pediatría (2003) Consumo de zumos de frutas y de debidas refrescantes por niños y adolescentes en España. Implicaciones para la salud de su mal uso y abuso. An Pediatr 58, 584-593.

11. Malik VS, Schulze MB \& Hu FB (2006) Intake of sugarsweetened beverages and weight gain: a systematic review. Am J Clin Nutr 84, 274-288.

12. Vartanian LR, Schwartz MB \& Brownell KD (2007) Effects of soft drink consumption on nutrition and health: a systematic review and meta-analysis. Am J Public Health 97, 667-675.

13. Gibson S (2008) Sugar-sweetened soft drinks and obesity: a systematic review of the evidence from observational studies and interventions. Nutr Res Rev 21, 134-147.

14. Olsen NJ \& Heitmann BL (2009) Intake of calorically sweetened beverages and obesity. Obes Rev 10, 68-75.

15. Chen L, Appel LJ, Loria C et al. (2009) Reduction in consumption of sugar-sweetened beverages is associated with weight loss: the PREMIER trial. Am J Clin Nutr 89, 1299-1306.

16. Heitmann BL \& Lissner L (1995) Dietary underreporting by obese individuals - is it specific or non-specific? BMJ 311, 986-989.

17. Lissner L (2002) Measuring food intake in studies of obesity. Public Health Nutr 5, 889-892.

18. Black AE, Coward WA, Cole TJ et al. (1996) Human energy expenditure in affluent societies: an analysis of 574 doublylabelled water measurements. Eur J Clin Nutr 50, 72-92. 
19. Schröder H, Covas MI, Marrugat J et al. (2001) Use of a three-day estimated food record, a 72-hour recall and a food-frequency questionnaire for dietary assessment in a Mediterranean Spanish population. Clin Nutr 20, 429-437.

20. Schofield WN (1985) Predicting basal metabolic rate, new standards and review of previous work. Hum Nutr Clin Nutr 39, Suppl. 1, 5-41.

21. Elosua R, Garcia M, Aguilar A et al. (2000) Validation of the Minnesota Leisure Time Physical Activity Questionnaire In Spanish Women. Investigators of the MARATDON Group. Med Sci Sports Exerc 32, 1431-1437.

22. Elosua R, Marrugat J, Molina L et al. (1994) Validation of the Minnesota Leisure Time Physical Activity Questionnaire in Spanish men. Am J Epidemiol 139, 1197-1209.

23. Guthrie JF \& Morton JF (2000) Food sources of added sweeteners in the diets of Americans. J Am Diet Assoc 100, 43-51.

24. Popkin BM, Armstrong LE, Bray GM et al. (2006) A new proposed guidance system for beverage consumption in the United States. Am J Clin Nutr 83, 529-542.

25. Giugliano D \& Esposito K (2008) Mediterranean diet and metabolic diseases. Curr Opin Lipidol 19, 63-68.
26. Muñoz MA, Fíto M, Marrugat J et al. (2009) Adherence to the Mediterranean diet is associated with better mental and physical health. Br J Nutr 101, 1821-1827.

27. Martinez-Gonzalez MA, Bes-Rastrollo M, Serra-Majem L et al. (2009) Mediterranean food pattern and the primary prevention of chronic disease: recent developments. Nutr Rev 67, Suppl. 1, S111-S116.

28. Ballew C, Kuester S \& Gillespie C (2000) Beverage choices affect adequacy of children's nutrient intakes. Arch Pediatr Adolesc Med 154, 1148-1152.

29. Yamada M, Murakami K, Sasaki S et al. (2008) Soft drink intake is associated with diet quality even among young Japanese women with low soft drink intake. J Am Diet Assoc 108, 1997-2004.

30. Bes-Rastrollo M, Sánchez-Villegas A, Gómez-Gracia E et al. (2006) Predictors of weight gain in a Mediterranean cohort: the Seguimiento Universidad de Navarra Study. Am J Clin Nutr 83, 362-370.

31. Bawa $S$ (2005) The role of the consumption of beverages in the obesity epidemic. $J R$ Soc Promot Health 125, 124-128.

32. Bachman CM, Baranowski T \& Nicklas TA (2006) Is there an association between sweetened beverages and adiposity? Nutr Rev 64, 153-174. 